\title{
Observation of the generation of stacking faults and active degradation measurements on off-axis and on-axis $4 \mathrm{H}-\mathrm{SiC}$ PiN diodes
}

\author{
N. Thierry-Jebali, 1, a) J. Hassan, ${ }^{2}$ M. Lazar, ${ }^{1}$ D. Planson, ${ }^{1}$ E. Bano, ${ }^{3}$ A. Henry, ${ }^{2}$ E. \\ Janzén, ${ }^{2}$ and P. Brosselard ${ }^{1}$,b) \\ 1) Université de Lyon, INSA de Lyon, Laboratoire Ampère, \\ 21 av. Jean Capelle F69621 Villeurbanne, France \\ 2) Department of Physics, Chemistry and Biology, Linköping University, \\ SE-581 83 Linköping, Sweden \\ 3) Grenoble INP, Laboratoire IMEP, 3 rue Paris Parvis Lois Nel BP 257 F38016 \\ Grenoble, France
}

(Dated: 7 November 2012)

PiN diodes have been fabricated on nominally on-axis Si-face $4 \mathrm{H}-\mathrm{SiC}$ material and their electrical characteristics are compared to PiN diodes processed with exactly the same device process recipe on 8-off $4 \mathrm{H}-\mathrm{SiC}$ material. Some diodes had an optical window on the top metal contact to observe the possible Stacking Faults generation and motion with Photo Emission Microscopy. The diodes were electrically characterized in forward voltage to test their stability. Electrical characterizations demonstrate that there is no noticeable degradation for the diodes processed on on-axis $4 \mathrm{H}-\mathrm{SiC}$ substrate and with optical characterization the formation of stacking faults was not observed.

a) nicolas.thierry-jebali@insa-lyon.fr

b) pierre.brosselard@insa-lyon.fr 
$\mathrm{SiC}$ is one of the most promising and studied semiconductor for high voltage, high temperature and high frequency devices due to its exceptional properties like a wide band gap, a high breakdown electric field, a high electron saturation velocity and a high thermal conductivity.

In the $1200 \mathrm{~V}$ range, unipolar devices such as Junction Barrier Schottky (JBS) and Vertical Junction Field Effect Transistor ( VJFET) are commercially available and currently used to provide high power converters. For higher voltage $(>3000 \mathrm{~V})$, bipolar devices are needed to preserve reasonably low series resistance. However, fabricating efficient SiC bipolar power devices is quite difficult. Indeed, a positive bias on a P-N junction generates the formation and motion of Shockley Stacking Faults (SFs) ${ }^{1}$.

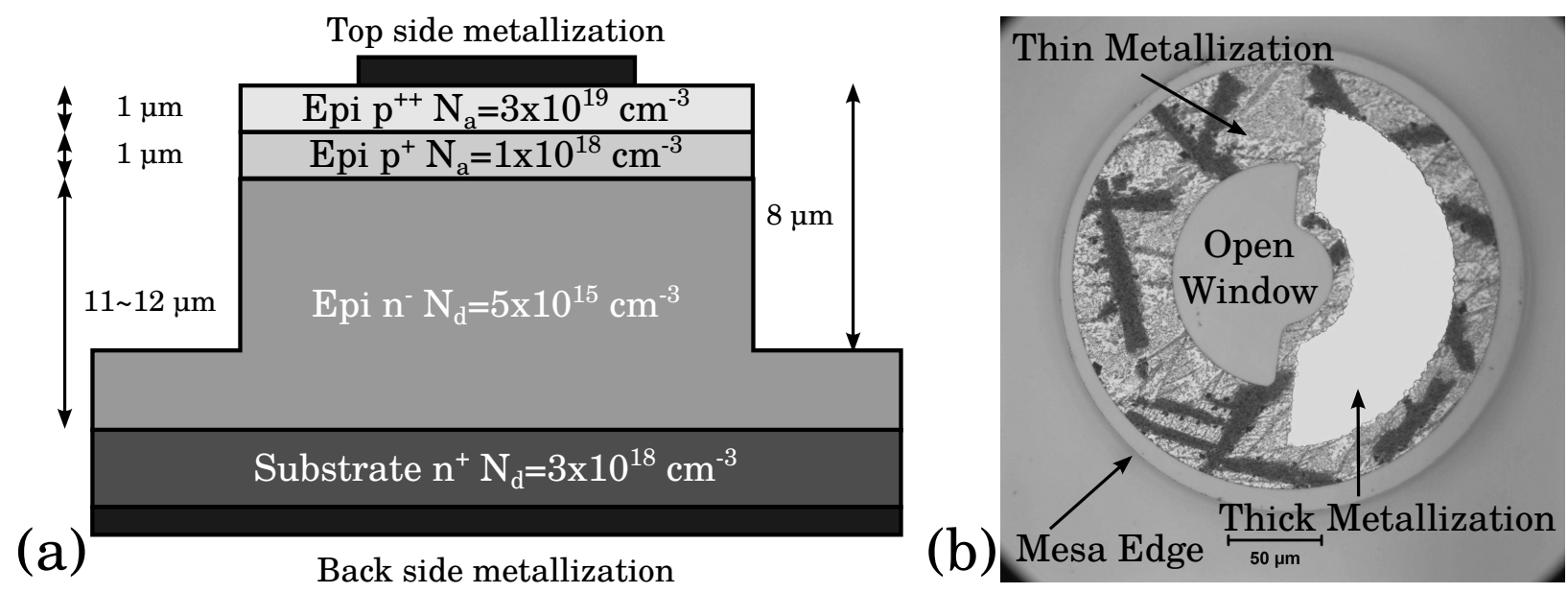

FIG. 1. a) Schematic cross-section of the fabricated diode and b) top-view of the studied diodes.

It has been established that SFs induce energy levels in the $\mathrm{SiC}$ band-gap leading to reduce the electronic minority carrier lifetime and generate the so called "forward voltage drift" phenomenon ${ }^{1,2}$. Such a phenomenon is critical as it deteriorates the differential resistance to breakdown voltage trade-off, increases the conduction power losses and leads to the device overheating.

The degradation of bipolar structures was initially mentioned during the ECSCRM 2000 conference in Germany ${ }^{2,3}$ and since many groups have tried to explain the origin, the mechanisms and propagation of such phenomenon ${ }^{1}$. Currently, the generation and motion of SFs during forward bias are proposed to be due to the change of Basal Plane Dislocation (BPD) into SF. The identification of driving force of the fault expansion remains unknown 1 .

The key to prevent degradation of PiN diodes is to decrease the BPDs density by an 
improvement of $4 \mathrm{H}-\mathrm{SiC}$ material quality. Some issues have been proposed on off-cut substrates by different groups $\underline{-6}$ - and are currently used for the commercial material fabrication. However, these enhancements reduce the growth parameter windows and lead to increase the density of other defects like carrots or ingrown stacking faults 1 .

On-axis homoepitaxial growth on $4 \mathrm{H}-\mathrm{SiC}$ substrates has been shown to completely eliminate for a 2 inch on-axis $4 \mathrm{H}-\mathrm{SiC}$ wafer the replication of the basal plane dislocations from the substrate into the epilayer ${ }^{7}$. In addition, no material will be wasted if the wafers are cut from crystal ingots grown on on-axis seeds. Thus, nominally on-axis material could be another key to the production of reliable $\mathrm{SiC}$ bipolar devices. Preliminary results have showed stable forward voltage in bipolar devices made on on-axis epilayers ${ }^{8}, 9$, however the forward voltage was abnormally high (almost $8 \mathrm{~V}$ with a current density higher than 100 A. $\left.\mathrm{cm}^{-2}\right)$.

In this work, we compare PiN diodes fabricated on 8-off and on-axis 4H-SiC substrate characterized by electrical measurement and Photon Emission Microscopy (PEM).

Homoepitaxial growth has been performed in a hot-wall CVD reactor using on-axis and off-axis $4 \mathrm{H}-\mathrm{SiC}$ substrates. The $\mathrm{Si}$ and $\mathrm{C}$ sources were silane and propane with a mixture of hydrogen and argon as a carrier gas. Since the in-situ surface preparation and ${ }^{10}$ growth processes $^{7}$ are different for on- and off-axis homoepitaxial growth, layers on both substrates were grown separately but with a similar $\mathrm{N}^{-} / \mathrm{P}^{+} / \mathrm{P}^{++}$epilayer structure, both in terms of thickness $(12 \mu \mathrm{m} / 1 \mu \mathrm{m} / 1 \mu \mathrm{m})$ and doping concentrations $\left(5 \times 10^{15} \mathrm{~cm}^{-3} / 8 \times 10^{17}\right.$ $\left.\mathrm{cm}^{-3} / 5 \times 10^{19} \mathrm{~cm}^{-3}\right)$, as shown in FIG. 1 $\mathrm{ra}$. The doping level and the thickness of the drift epilayer correspond to a $1200 \mathrm{~V}$ technology. Indeed, the purpose of this work is to optimize and analyze the active area on a cheapest $\mathrm{N}^{-}$epilayer than a high blocking voltage technology $(>3.3 \mathrm{kV})$. The prior preparation of epitaxial layers are the same as thicker $\mathrm{N}^{-}$layer. A mirror like surface morphology has been observed for the epilayers grown on off-cut substrates. However, on-axis epilayers show comparatively an inhomogeneous and rough surface owing to different epitaxial growth mechanisms ${ }^{7}$. This roughness is characterized by terraces with steps which can be as high as $2-3 \mu \mathrm{m}$.

In order to minimize defects in the semiconductor, we decided to perform an ion implantation free structure. The anode periphery has been defined to be an $8 \mu \mathrm{m}$ depth mesa termination. The process parameters of the fabricated diodes are better described elsewhere ${ }^{11}$. FIG. 1-b exhibits a top view of a diode fabricated for the Photon Emission 

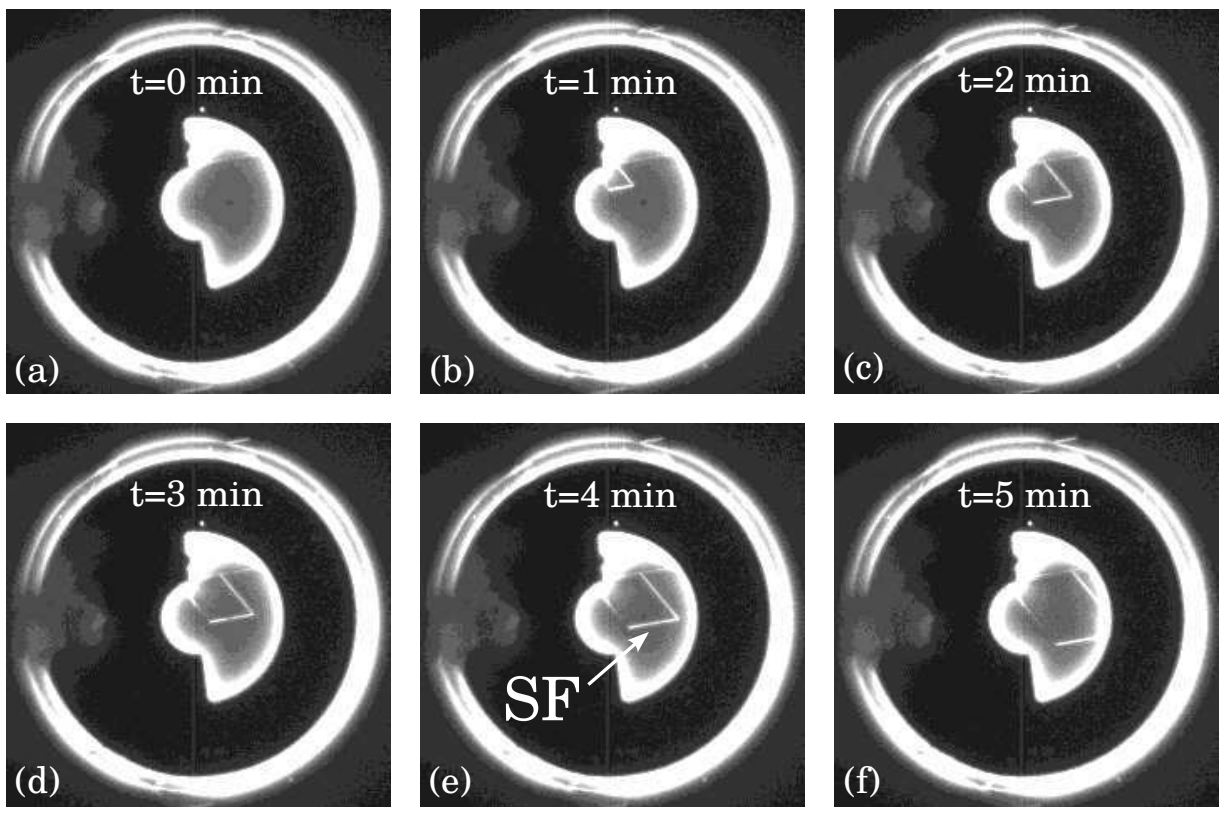

FIG. 2. PEM pictures of the $8^{\circ}$-off sample at a $3.5 \mathrm{~V}$ bias $\left(J \approx 400 A . \mathrm{cm}^{-2}\right)$ for a time of a) $\mathrm{t}=0$ $\min , \mathrm{b}) \mathrm{t}=1 \min , \mathrm{c}) \mathrm{t}=2 \min , \mathrm{d}) \mathrm{t}=3 \min \mathrm{e}) \mathrm{t}=4 \min , \mathrm{f}) \mathrm{t}=5 \min$.
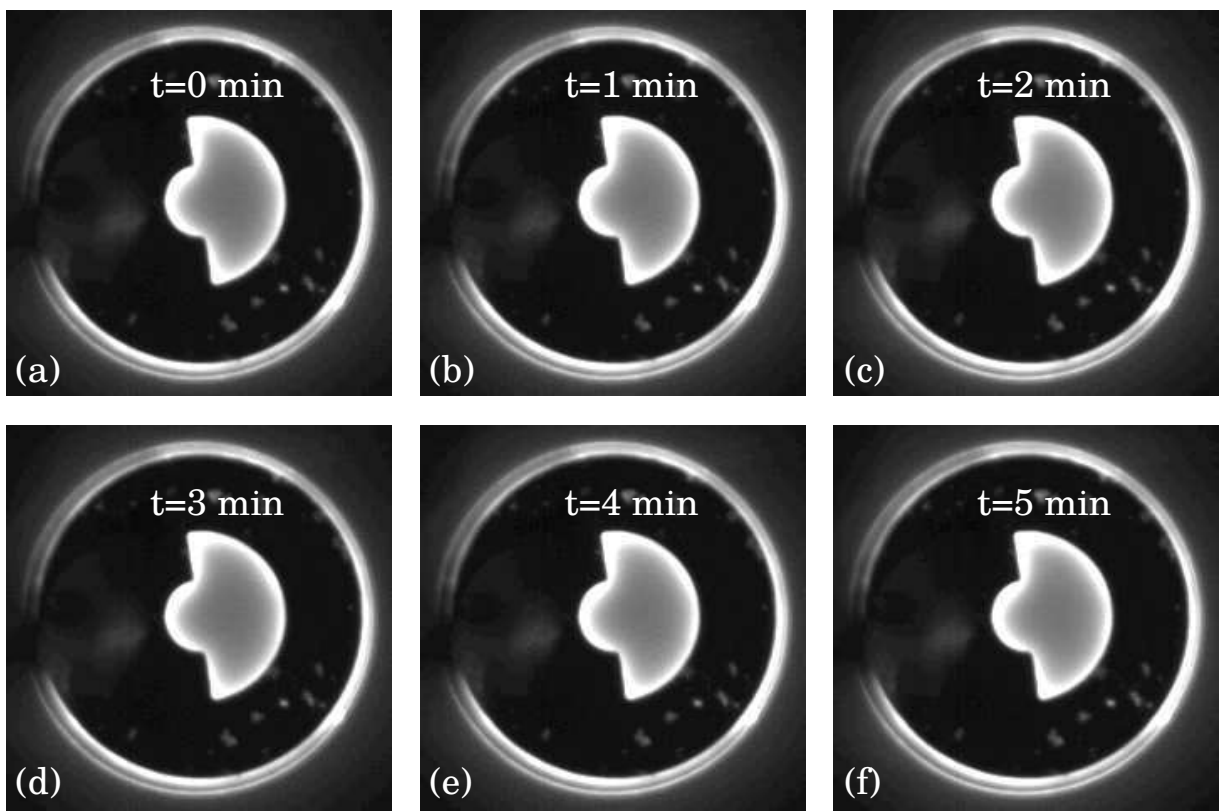

FIG. 3. PEM pictures of the on-axis sample at a $3.5 \mathrm{~V}$ bias $\left(J \approx 200 \mathrm{~A} . \mathrm{cm}^{-2}\right)$ for a time of a) $\mathrm{t}=0$ $\min , \mathrm{b}) \mathrm{t}=1 \mathrm{~min}, \mathrm{c}) \mathrm{t}=2 \mathrm{~min}$.

Microscopy (PEM) observation. The anode is a $250 \mu \mathrm{m}$ diameter circle including an open window which allows the observation of SFs generation and their motion monitored by 
PEM. Circular diodes with a diameter of $400 \mu \mathrm{m}$ have been used for active degradation measurement.

PEM pictures have been obtained by using a Mitutoyo FS50Y microscope and a Hamamatsu C4880 CCD sensor $\underline{\underline{12}}$. The CCD sensor has been cooled at $-55^{\circ} \mathrm{C}$ in order to minimize the thermal noise. Voltage stress have been performed using a HP 4145 in "2 wires" configuration during the PEM observations at 2.8, 3, 3.2 and $3.5 \mathrm{~V}$ during 5 min. In order to characterize the possible SFs generation and their motion, PEM pictures have been taken every minute. A minimum of 10 diodes have been characterized per sample set.

Active degradation has been achieved by applying a current stress at $J=10 \mathrm{A.cm}{ }^{-2}$ and

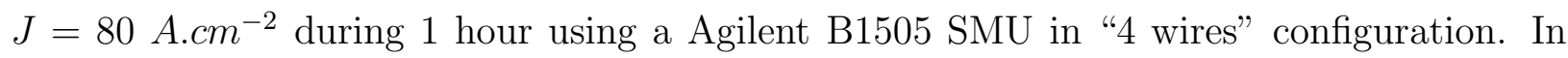
order to get a better view of the evolution of the measured voltage as a function of time the evolution of $\frac{\left(V-V_{t 0}\right)}{V_{t 0}}$ are plotted where $V$ is the measured voltage and $V_{t 0}$ the initial voltage value at $t=0 \mathrm{~s}$.

Only a few representative PEM pictures are reported in this paper for a better readability. FIG. 22 and FIG. [3] show PEM pictures for a voltage bias of 3.5 V for the off-cut and on-axis samples respectively and the results on active degradation are reported on FIG. 4.

FIG. 2 exhibits the generation and the motion of a classical rhombohedral-shaped SF when a positive bias is applied. This phenomenon is also observed for lower voltage as established by other groups 1 . The generation and motion of SFs have been identified for each characterized diodes on off-cut substrate. However, the density of SFs varies dramatically and its value is randomly distributed on the sample.

In contrast, no SF generation has been detected in any of the diodes made on on-axis material characterized by PEM observation whatever the voltage bias (FIG. 3). The only photo-emission observed in this case is due to the $\mathrm{Al}$ level / conduction band transition. These observations are promising for getting non degenerative PiN diodes.

FIG. 4 4 a and -b display the evolution of the variation of the measured voltage value with the initial measured value as a function of time at various current densities for different diodes, $J=10 \mathrm{~A} \cdot \mathrm{cm}^{-2}$ (diodes "1" and "2") and $J=80 \mathrm{~A} \cdot \mathrm{cm}^{-2}$ (diode " 3 ") for the off-cut substrate and the on-axis substrate, respectively. The applied current density $J$ and the $V_{t 0}$ value for each value are reported on TAB. II. These values are in agreement with a bipolar current conduction.

One can see on FIG. 4-a an increase of the differential resistance during the first hundreds 

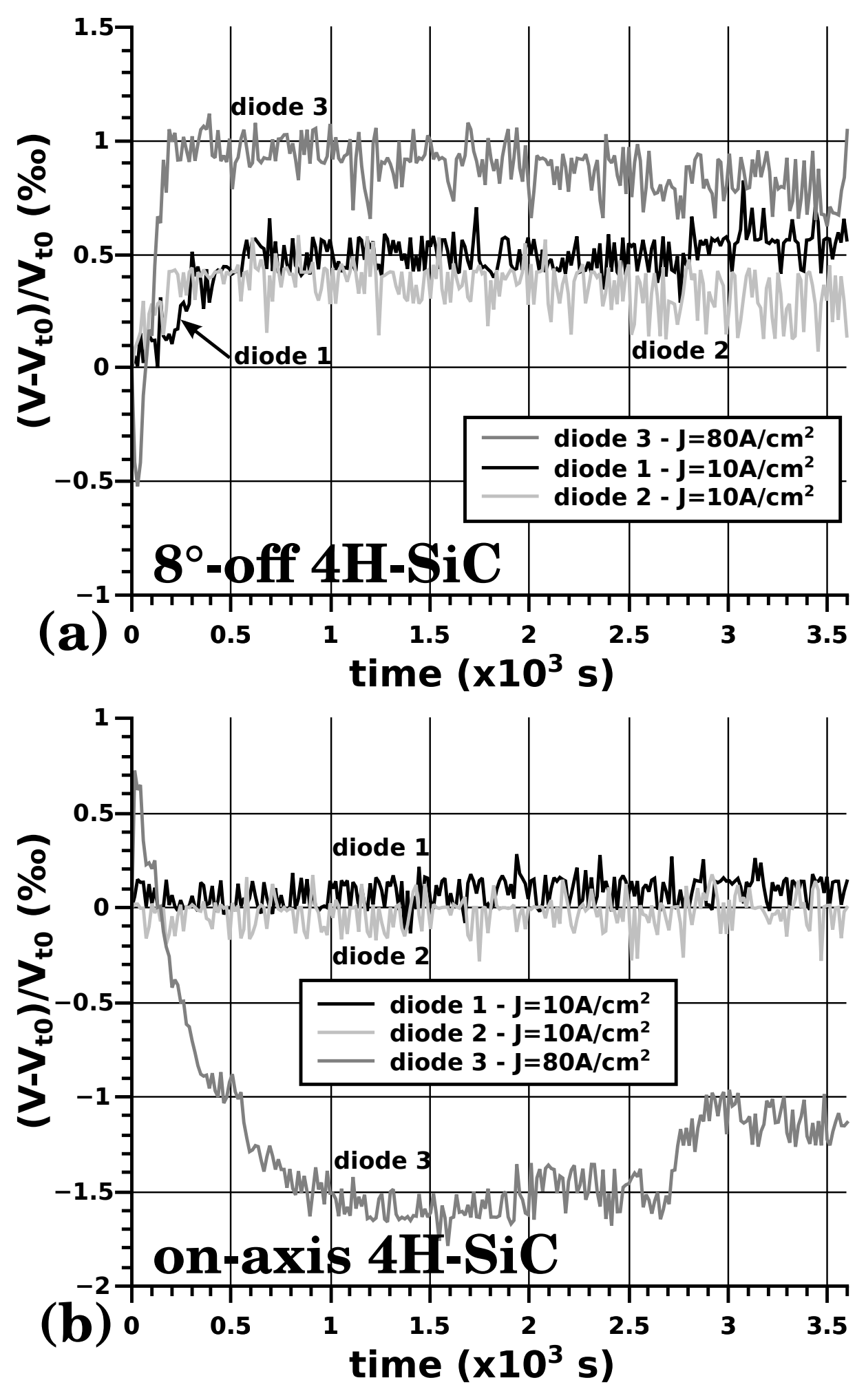

FIG. 4. Evolution of forward voltage during active degradation with different current density for a) $8^{\circ}$-off samples, b) on-axis samples

of seconds during the polarization at $J=10 \mathrm{A.cm} \mathrm{cm}^{-2}$ for the $8^{\circ}$-off sample. This increase is 
TABLE I. Measured values of $V_{t 0}$ for each diodes reported on FIG. 4 for $8^{\circ}$ off and on-axis sample during the active degradation at $J=10 \mathrm{~A} . \mathrm{cm}^{-2}$ and $J=80 \mathrm{A.cm}^{-2}$

\begin{tabular}{ccccccc}
\multicolumn{3}{c}{$8^{\circ}$ off sample } & & \multicolumn{3}{c}{ on-axis sample } \\
\cline { 5 - 7 } Diode $\mathrm{N}^{\circ} J\left(A . \mathrm{cm}^{-2}\right)$ & $V_{t 0}(V)$ & & Diode $\mathrm{N}^{\circ} J\left(A . \mathrm{cm}^{-2}\right)$ & $V_{t 0}(V)$ \\
1 & 10 & 2.8408 & & 1 & 10 & 2.8776 \\
2 & 10 & 2.8372 & 2 & 10 & 2.868 \\
3 & 80 & 3.0476 & 3 & 80 & 3.18 \\
\hline
\end{tabular}

more relevant for a higher current density. Indeed the measured voltage increases at $0.5 \%$ o of $V_{t 0}$ at $J=10 \mathrm{~A} . \mathrm{cm}^{-2}$ and at $1 \%$ of $V_{t 0}$ at $J=80 \mathrm{~A} . \mathrm{cm}^{-2}$. This phenomenon agrees with the generation and motion of SFs observed by PEM.

As expected, the measured voltage of diodes processed on on-axis material polarized at $J=10 \mathrm{~A} . \mathrm{cm}^{-2}$ is stable during the whole experiment since no SF generation has occurred. Surprisingly, a decrease of the differential resistance is noted for a higher current density, so the static electrical properties are improved during the polarization. Currently we cannot explain from a physical point of view what append in our diodes during the polarization.

In this work, we have compared PiN diode fabricated on $8^{\circ}$-off and on-axis $4 \mathrm{H}-\mathrm{SiC}$ substrates. We have clearly identified the generation and motion of SFs during a forward voltage bias and characterized a "voltage drift" for diodes processed on off-cut substrate. In contrast, no degradation occurred for sample fabricated on on-axis substrate and static electrical properties are improved during the operation under high current stress.

In conclusion, this work tends to demonstrate that reliable high breakdown voltage PiN diodes could be achieved by using $4 \mathrm{H}-\mathrm{SiC}$ on-axis substrate.

\section{ACKNOWLEDGMENT}

The authors want to thank the Agence Nationale pour la Recherche, ANR, for its financial support in the framework of the Very High Voltage Devices SiC project (VHVD-SiC, ANR-08-BLAN-0191 ), the Swedish Energy Agency (project 32917-1), Swedish Research Council (VR 2009-3383) and Swedish Government Strategic Research Area Grant in Material Science. 


\section{REFERENCES}

${ }^{1}$ M. Skowronski and S. Ha, Journal of applied physics 99, 011101 (2006).

${ }^{2}$ J. Bergman, H. Lendenmann, P. Nilsson, U. Lindefelt, and P. Skytt, in Materials Science Forum, Vol. 353 (Trans Tech Publ, 2001) pp. 299-302.

${ }^{3}$ H. Lendenmann, F. Dahlquist, N. Johansson, R. Söderholm, P. Nilsson, J. Bergman, and P. Skytt, in Materials Science Forum, Vol. 353 (Trans Tech Publ, 2001) pp. 727-730.

${ }^{4}$ J. Sumakeris, M. Das, M. McD. Hobgood, S. Müller, M. Paisley, S. Ha, M. Skowronski, J. Palmour, C. Carter Jr, in Materials Science Forum, Vol. 457 (Trans Tech Publ, 2004) pp. $1113-1116$.

${ }^{5}$ J. Sumakeris, J. Jenny, and A. Powell (Materials Research Society, 2005) pp. 280-286.

${ }^{6}$ J. Sumakeris, J. Bergman, M. Das, C. Hallin, B. Hull, E. Janzén, H. Lendenmann, M. O'Loughlin, M. Paisley, S. Ha, et al., in Materials science forum, Vol. 527 (Trans Tech Publ, 2006) pp. 141-146.

${ }^{7}$ J. Hassan, J. Bergman, A. Henry, and E. Janzén, Journal of Crystal Growth 310, 4424 (2008).

${ }^{8}$ J. Hassan, J. Bergman, A. Henry, P. Brosselard, P. Godignon, and E. Janzén, in Materials Science Forum, Vol. 615 (Trans Tech Publ, 2009) pp. 133-136.

${ }^{9}$ P. Bergman, J. Hassan, A. Ellison, A. Henry, P. Godignon, P. Brosselard, and E. Janzén, in MRS Proceedings, Vol. 1069 (Cambridge Univ Press, 2008).

${ }^{10}$ J. Hassan, J. Bergman, A. Henry, and E. Janzén, Journal of Crystal Growth 310, 4430 (2008).

${ }^{11}$ G. Civrac, F. Laariedh, N. Thierry Jebali, M. Lazar, D. Planson, P. Brosselard, J. Hassan, A. Henry, E. Janzén, B. Vergne, et al., in Materials Science Forum, Vol. 717 (Trans Tech Publ, 2012) pp. 969-972.

${ }^{12}$ C. Banc, E. Bano, T. Ouisse, K. Vassilevski, and K. Zekentes, in Materials Science Forum, Vol. 389 (Trans Tech Publ, 2002) pp. 1293-1296. 